\title{
How strong singularities can be regularized by logistic degradation in the Keller-Segel system?
}

\author{
Michael Winkler ${ }^{1}$
}

Received: 9 September 2018 / Accepted: 5 February 2019 / Published online: 15 February 2019 (c) Fondazione Annali di Matematica Pura ed Applicata and Springer-Verlag GmbH Germany, part of Springer Nature 2019

\section{Abstract}

The parabolic-elliptic version of the logistic Keller-Segel system given by

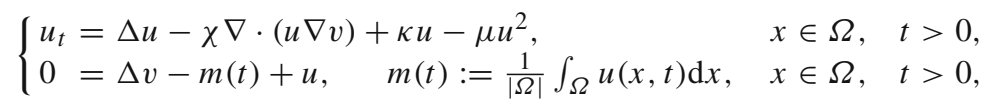

is considered in the ball $\Omega=B_{R}(0) \subset \mathbb{R}^{n}$ with $n \geq 1$ and $R>0$, and with parameters $\kappa \in \mathbb{R}, \chi>0$ and $\mu>0$. The focus is on the question how the zero-order dissipative term $-\mu u^{2}$ herein, forming the apparently most essential difference between $(\star)$ and the classical parabolic-elliptic Keller-Segel system, affects the evolution of supposedly present singular structures. For this purpose, a Neumann-type initial boundary value problem for $(\star)$ with $\mu>\chi$ is studied for radially decreasing nonnegative initial data $u_{0} \in C^{1}(\bar{\Omega} \backslash\{0\})$ fulfilling $u_{0}(x) \leq K \phi(|x|)$ for all $x \in \bar{\Omega} \backslash\{0\}$ with some $K>0$ and some function $\phi:(0, \infty) \rightarrow$ $[1, \infty)$ which, besides some technical assumptions, complies with the key condition

$$
\int_{0}^{1} r^{n-1} \ln \phi(r) \mathrm{d} r<\infty .
$$

It is seen that for this class of data, including any such $u_{0}$ satisfying

$$
u_{0}(x) \leq K e^{\lambda|x|^{-\alpha}} \quad \text { for all } x \in \bar{\Omega} \backslash\{0\}
$$

with some positive constants $K, \lambda$ and $\alpha<n$, the problem in question in fact admits a global solution $(u, v)$ which is smooth and classical in $\bar{\Omega} \times(0, \infty)$ and attains the initial data in the topology of $C_{\text {loc }}^{0}(\bar{\Omega} \backslash\{0\})$ as $t \searrow 0$. In view of the well-known fact that in the unperturbed Keller-Segel system already some finite-mass Radon measure-type singularities give rise to persistently singular solutions, and that hence no significant smoothing action can be expected there when infinite-mass distributions are initially present, these results reveal that zero-order quadratic degradation indeed may have a substantial effect on cross-diffusive interaction by enforcing instantaneous smoothing even of initial data exhibiting some exponentially strong singularities.

$\bowtie \quad$ Michael Winkler

michael.winkler@math.uni-paderborn.de

1 Institut für Mathematik, Universität Paderborn, 33098 Paderborn, Germany 
Keywords Chemotaxis $\cdot$ Logistic source $\cdot$ Singularity $\cdot$ Instantaneous smoothing

Mathematics Subject Classification Primary 35B65; Secondary 92C17 · 35Q92 - 35K55 . 35B40

\section{Introduction}

Understanding chemotaxis-driven dynamics of population distributions far from spatial homogeneity is forming a recurrent theme in several disciplines from biology and especially in corresponding theoretical studies [30]. Stimulated by findings on spontaneous formation of aggregates and, more generally, on various facets of complex collective behavior in chemotactically migrating populations when distributed in a strongly heterogeneous manner, substantial efforts in mathematical analysis have led to considerable insight into numerous qualitative aspects related to the destabilizing potential of taxis-type cross-diffusion, as constituting the apparently most characteristic common ingredient not only in the celebrated Keller-Segel model for such processes [18], in its simplest form reducing to the parabolic system

$$
\left\{\begin{array}{l}
u_{t}=\Delta u-\nabla \cdot(u \nabla v), \\
v_{t}=\Delta v-v+u,
\end{array}\right.
$$

for the population density $u=u(x, t)$ and the chemoattractant concentration $v=v(x, t)$, but beyond this also in a large class of relatives thereof $[15,30]$. Here the knowledge seems most comprehensive with regard to the question under which circumstances solutions may develop singularities either in finite or infinite time. In the context of (1.1), for instance, it is known that such unboundedness phenomena do occur in spatially two- or higher-dimensional settings $[14,42]$, but that any such explosion is ruled out in the associated one-dimensional version [29]; in fact, a large literature has revealed remarkably detailed information on corresponding dichotomies in several refinements and derivates of $(1.1)$ [8,12,23,27,36,40,46], in its most delicate part, namely arguments concerned with blowup detection, typically relying on certain simplifications in which the chemoattractant evolution is governed by an elliptic equation $[3,16,22,26,37,47]$.

In comparison to this, much less seems known with regard to the question how chemotactic evolution copes with locally extreme population concentrations in the sense of singularities which are supposedly present at some instant. Here adaptation and refinement of straightforward functional analytical approaches, e.g., based on transformation to integral equations via Duhamel formulae, and on design of suitable fixed point frameworks, have made it possible to construct certain generalized local-in-time solutions, instantaneously becoming smooth and classical, for initial data with quite low integrability properties. In the prototypical setup of the Neumann problem for (1.1) in bounded domains $\Omega \subset \mathbb{R}^{n}, n \geq 2$, for instance, immediate regularization in this sense has been shown to occurs for all initial data $\left(u_{0}, v_{0}\right)$ from $L^{1}(\Omega) \times W^{1, n}(\Omega)$, and actually even for slightly larger classes involving either Radon measure-type distributions in the first component when $n=2$, or suitable Morrey spaces in both components when $n \geq 3$ [4,5,33]; requirements of this flavor apparently cannot be substantially relaxed even when beyond this, more subtle use is made of a priori estimates implied from the well-known gradient-flow structure of the particular cross-diffusive interaction in (1.1) [6,7]. This is further supported by observations indicating absence of smoothing in presence of initial data close to the above spaces: In some two-dimensional Keller-Segel systems, namely, some finite-mass but measure-valued cell distributions are known to evolve 
into persistent measure-type singularities ([13,24]; cf. also [2,34]); in the case $n \geq 3$, the stationary singularities becoming manifest in the so-called Chandrasekhar solutions to a simplified parabolic-elliptic variant of (1.1), as determined by $u(x, t):=\frac{2(n-2)}{|x|^{2}}, x \neq 0$, even provide explicit examples that strongly indicate criticality of spatial $L^{\frac{n}{2}}$ spaces as regards the possibility of instantaneous regularization.

\subsection{Main results: detecting additional regularization effects of logistic dampening}

The purpose of the present study is to investigate how far the evolution of singular structures may be affected by the presence of logistic-type growth restrictions. In fact, a large literature suggests that accordingly obtained logistic Keller-Segel systems can be viewed as a natural first step to adapt the prototypical and hence quite simple model (1.1) so as to account for mechanisms of competition-induced overcrowding prevention which seem virtually ubiquitous in numerous situations of biological relevance (see, e.g., [11,35,39] or [32] for some examples, or also [15,30] for a broader overview). In fact, several precedents have been indicating that on the way from (1.1) to fully realistic models, despite their increased complexity and especially their apparent lack of appropriate energy structures, such logistic chemotaxis systems remain accessible to a considerably large variety of mathematical tools; accordingly, not only quite comprehensive conclusions are available in the fields of global classical solvability for smooth data [28,38,41,48], of constructing attractors [1,28,29], and of proving asymptotic homogenization in cases of strong dampening [9,43] (cf. also [21]), but beyond this even some facets of the rich dynamics of such systems, as reported by numerical findings [31], could be captured by some rigorous results on emergence of extremely large cell densities at possibly intermediate time scales [17,20,44,45].

In contract to this, the knowledge with regard to the smoothing potential of logistic death terms when confronted with locally large distributions seems quite thin; after all, the apparently furthest reaching explicitly formulated results in this respect, addressing cases of $L^{2}$ initial values in two-dimensional domains [28], seem to allow extension, through adaptation of the arguments, e.g., in [5], at least to $L^{\frac{n}{2}}$ data in general $n$-dimensional settings. To our impression, however, covering classes of initial data substantially larger than those known to be admissible already for (1.1) has nowhere been achieved so far. Our main results in this direction now identify a genuine supplementary smoothing action of quadratic degradation, as forming the essential additional ingredient in logistic Keller-Segel systems when compared to (1.1). Indeed, we shall see that in such systems instantaneous smoothing may in fact occur for initial data exhibiting singular behavior much more extreme than power-type, even up to some exponential strength, and thus far below levels of integrability.

To make this more precise, let us resort to a setting as simple as possible but yet potentially capturing the main characteristics of the problem context under consideration, and hence consider radially symmetric solutions to the parabolic-elliptic problem

$$
\begin{cases}u_{t}=\Delta u-\chi \nabla \cdot(u \nabla v)+\kappa u-\mu u^{2}, & x \in \Omega, \quad t>0, \\ 0=\Delta v-m(t)+u, & x \in \Omega, \quad t>0, \\ \frac{\partial u}{\partial v}=\frac{\partial v}{\partial v}=0, & x \in \partial \Omega, \quad t>0, \\ u(\cdot, 0)=u_{0}(x), & x \in \Omega,\end{cases}
$$

in the ball $\Omega=B_{R}(0) \subset \mathbb{R}^{n}$ with $n \geq 1$ and $R>0$, where $\chi>0, \kappa \in \mathbb{R}, \mu>0$ and

$$
m(t):=\frac{1}{|\Omega|} \int_{\Omega} u(x, t) \mathrm{d} x, \quad t>0 .
$$


Our standing assumption on the initial data will be that

$$
\begin{cases}u_{0} \in C^{1}(\bar{\Omega} \backslash\{0\}) & \text { is nonnegative and radially symmetric } \\ & \text { and nonincreasing with respect to }|x| \in(0, R],\end{cases}
$$

and in most places we will furthermore suppose that

$$
u_{0}(r) \leq K \phi(r) \quad \text { for all } r \in(0, R),
$$

where

$$
\phi \in C^{2}((0, \infty)) \text { is such that } \phi \geq 1 \text { and } \phi^{\prime}<0 \text {, and that } \phi(r) \nearrow+\infty \text { as } r \searrow 0 \text {. }
$$

Here and throughout the sequel, whenever this appears convenient and without any risk to cause confusion, we shall tacitly switch to the usual notation using radial variables, thus $a d$ lib replacing, e.g., $u_{0}(x)$ with $u_{0}(r)$ for $r=|x|$.

Now our main results will require that the singular behavior of $\phi$, and hence of $u_{0}$, can be controlled in such a way that besides the technical but otherwise comparatively mild hypotheses that

$$
\phi^{\prime \prime}(r) \leq K_{1} \phi^{2}(r) \quad \text { for all } r \in(0,1)
$$

and that

$$
\phi(2 r) \leq K_{2} r^{n} \phi(r) \quad \text { for all } r \in(0,1),
$$

the crucial logarithmic integrability condition

$$
\int_{0}^{1} r^{n-1} \ln \phi(r) \mathrm{d} r<\infty
$$

is satisfied. In this general framework, we shall obtain the following statement on global existence of solutions which immediately become smooth and classical, and which attain the prescribed and possibly singular initial trace locally uniformly outside the spatial origin:

Theorem 1.1 Let $\Omega=B_{R}(0) \subset \mathbb{R}^{n}$ with some $n \geq 1$ and $R>0$, and let $\kappa \in \mathbb{R}, \chi>0$ and $\mu>0$ be such that

$$
\mu>\chi \text {. }
$$

Moreover, assume that $u_{0}$ satisfies (1.4) and (1.5) with some $K>0$ and some function $\phi:(0, \infty) \rightarrow \mathbb{R}$ for which there exist $K_{1}>0$ and $K_{2}>0$ such that (1.6)-(1.9) hold. Then one can find a pair $(u, v)$ of nonnegative radially symmetric functions

$$
\left\{\begin{array}{l}
u \in C^{0}((\bar{\Omega} \backslash\{0\}) \times[0, \infty)) \cap C^{2,1}(\bar{\Omega} \times(0, \infty)) \quad \text { and } \\
v \in C^{2,0}(\bar{\Omega} \times(0, \infty))
\end{array}\right.
$$

which solves the boundary value problem in (1.2) in the classical sense in $\bar{\Omega} \times(0, \infty)$, for which the total mass functional enjoys the integrability property

$$
\int_{0}^{\mathrm{T}} \int_{\Omega} u(x, t) \mathrm{d} x<\infty \quad \text { for all } T>0,
$$

and which is such that furthermore

$$
u(\cdot, t) \rightarrow u_{0} \quad \text { in } C_{\mathrm{loc}}^{0}(\bar{\Omega} \backslash\{0\}) \quad \text { as } t \searrow 0 .
$$

When specified to the particular choice of functions $\phi$ representing exponential singularities, this directly implies the following concrete consequence. 
Corollary 1.2 Let $n \geq 1, R>0$ and $\Omega=B_{R}(0) \subset \mathbb{R}^{n}$, let $\kappa \in \mathbb{R}, \chi>0$ and $\mu>\chi$, and suppose that $u_{0}$ satisfies (1.4) as well as

$$
u_{0}(r) \leq K e^{\lambda r^{-\alpha}} \quad \text { for all } r \in(0, R)
$$

with some positive constants $K, \lambda$ and $\alpha$ such that

$$
\alpha<n \text {. }
$$

Then there exists a pair $(u, v)$ of functions for which the conclusion from Theorem 1.1 holds.

\subsection{Main ideas}

As thanks to the comparatively simple parabolic-elliptic structure of (1.2), assumption (1.10) can be seen to entail a pointwise upper bound for $\frac{t}{t+1} u$ by means of a comparison argument (Lemma 2.2), constructing a smooth limit in $\bar{\Omega} \times(0, \infty)$ of solutions to an appropriately regularized problem can be achieved by quite well-established bootstrap and compactness reasonings (Lemmas 2.6, 2.7). The main challenge will thus consist in asserting the claimed behavior of this solution to the boundary value problem in (1.2), and it turns out that for this it is not only sufficient but also essentially necessary (see Proposition 3.1) to control the asymptotic behavior of the nonlocal ingredient $m(t)$ in (1.2), and hence of the total mass functional $\int_{\Omega} u(x, t) \mathrm{d} x$, in the sense of time integrability near $t=0$ as in (1.12). This will be achieved on the basis of a second comparison argument, which will rely on the fact that thanks to (1.7) large multiples of $\phi$ become stationary supersolutions to an equivalent version of the first equation in (1.2) when restricted to radially nonincreasing functions $u$ and $v$ [see Lemma 3.2 and (2.8)], which forms the major motivation for the initial monotonicity, as assumed in (1.4) and fortunately inherited not only by $u(\cdot, t)$ but also by $v(\cdot, t)$ for $t>0$ (Lemmas 2.4, 2.5).

Since (1.8) and (1.9) guarantee that a combination of the above two pointwise inequalities for $u$ entails an estimate of the mass functional against an integrable function of time (Lemmas 3.3,3.4), through the second equation in (1.2) thus having at hand suitable bounds for $\nabla v$, yet $L^{1}$ with respect to time, we will firstly conclude that the initial trace is attained in the topology of $\left.W_{0}^{2,1}\left(\Omega \backslash \bar{B}_{\delta}(0)\right)\right)^{\star}$ for each $\delta \in(0, R)$ (Lemma 3.7). Secondly, this $L^{1}$ information on $\nabla v$ will enable us to derive precompactness in $C_{\text {loc }}^{0}(\bar{\Omega} \backslash\{0\})$ of $(u(\cdot, t))_{t \in(0,1)}$ and hence imply the desired convergence property (1.13), through a two-step regularity argument (Lemmas 3.8, 3.9).

\section{Approximate problems and their limit behavior for $t>0$}

\subsection{A family of approximate problems and a fundamental pointwise estimate}

Let us introduce a convenient regularization of (1.2) through approximation of a given and possibly nonsmooth initial function $u_{0}$ by a family $\left(u_{0 \varepsilon}\right)_{\varepsilon \in(0,1)}$ of suitably smooth functions on $\bar{\Omega}$ in the sense that

$$
0 \not \equiv u_{0 \varepsilon} \in C^{2}(\bar{\Omega}) \text { is radially symmetric for all } \varepsilon \in(0,1),
$$

that, when expressed in the variable $r=|x| \in[0, R]$, their gradients $u_{0 \varepsilon r}$ satisfy

$$
u_{0 \varepsilon r}(r) \leq 0 \quad \text { for all } r \in[0, R] \text { and } \varepsilon \in(0,1)
$$


as well as the compatibility condition

$$
u_{0 \varepsilon r}(R)=0 \quad \text { for all } \varepsilon \in(0,1),
$$

and that $u_{0 \varepsilon}$ approaches $u_{0}$ in the sense that

$$
u_{0 \varepsilon} \leq u_{0}+1 \quad \text { in } \bar{\Omega} \backslash\{0\} \text { for all } \varepsilon \in(0,1)
$$

and

$$
u_{0 \varepsilon} \rightarrow u_{0} \quad \text { in } C_{\mathrm{loc}}^{1}(\bar{\Omega} \backslash\{0\}) \quad \text { as } \varepsilon \searrow 0 .
$$

Then for each $\varepsilon \in(0,1)$, well-established arguments [25,47] assert local solvability of the approximate version of (1.2) given by

$$
\begin{cases}u_{\varepsilon t}=\Delta u_{\varepsilon}-\chi \nabla \cdot\left(u_{\varepsilon} \nabla v_{\varepsilon}\right)+\kappa u_{\varepsilon}-\mu u_{\varepsilon}^{2} & x \in \Omega, \quad t>0 \\ 0=\Delta v_{\varepsilon}-m_{\varepsilon}(t)+u_{\varepsilon}, \quad m_{\varepsilon}(t):=\frac{1}{|\Omega|} \int_{\Omega} u_{\varepsilon}(\cdot, t), & x \in \Omega, \quad t>0 \\ \frac{\partial u_{\varepsilon}}{\partial v}=\frac{\partial v_{\varepsilon}}{\partial v}=0, & x \in \partial \Omega, \quad t>0 \\ u_{\varepsilon}(x, 0)=u_{0 \varepsilon}(x), & x \in \Omega,\end{cases}
$$

in the following sense:

Lemma 2.1 Let $\chi>0, \kappa \in \mathbb{R}$ and $\mu>0$, and suppose that $u_{0}$ and $u_{0 \varepsilon}$ satisfy (1.4) and (2.1)-(2.5). Then for all $\varepsilon \in(0,1)$ there exist $T_{\max , \varepsilon} \in(0, \infty]$ and a uniquely determined classical solution $\left(u_{\varepsilon}, v_{\varepsilon}\right) \in\left(C^{0}(\bar{\Omega} \times[0, \infty)) \cap C^{2,1}(\bar{\Omega} \times(0, \infty))\right)^{2}$ such that $u_{\varepsilon}(\cdot, t)$ and $v_{\varepsilon}(\cdot, t)$ are positive and radially symmetric in $\bar{\Omega}$ for all $t>0$, and that

$$
\text { if } T_{\max , \varepsilon}<\infty \text {, then } \limsup _{t \nearrow T}\left\|u_{\varepsilon}(\cdot, t)\right\|_{L^{\infty}(\Omega)}=\infty .
$$

For later reference in several places, let us observe on combining the first two equations in (2.6) that $u_{\varepsilon}$ actually solves the Neumann problem for the scalar parabolic equation

$$
u_{\varepsilon t}=\Delta u_{\varepsilon}-\chi \nabla u_{\varepsilon} \cdot \nabla v_{\varepsilon}-\chi m_{\varepsilon}(t) u_{\varepsilon}+\kappa u_{\varepsilon}-(\mu-\chi) u_{\varepsilon}^{2}, \quad x \in \Omega, \quad t \in\left(0, T_{\max , \varepsilon}\right),
$$

with the coefficient function $\nabla v_{\varepsilon}$ in fact forming a nonlocal ingredient due to its dependence on $u_{\varepsilon}$ through the second equation in (2.6).

Here with regard to our overall goal of achieving regularization, the rightmost summand appears to be favorable if $\chi$ and $\mu$ comply with the hypotheses from Theorem 1.1. Throughout the sequel, we shall accordingly assume that

$$
\mu>\chi,
$$

and then may draw a first but substantial conclusion thereof through parabolic comparison with spatially flat functions as follows.

Lemma 2.2 Let $\kappa \in \mathbb{R}$ and $0<\chi<\mu$, and suppose that (1.4) holds. Then for each $\varepsilon \in(0,1)$, the solution $\left(u_{\varepsilon}, v_{\varepsilon}\right)$ of $(2.6)$ is global in time and satisfies

$$
u_{\varepsilon}(x, t) \leq \frac{1}{(\mu-\chi) t}+\frac{\kappa_{+}}{\mu-\chi} \quad \text { for all } x \in \Omega \text { and } t>0 .
$$

Proof For fixed $\varepsilon \in(0,1)$ we may use the regularity statement in Lemma 2.1 to pick $c_{1}(\varepsilon)>$ 0 such that

$$
u_{\varepsilon}(x, t) \leq c_{1}(\varepsilon) \quad \text { for all } x \in \Omega \text { and } t \in\left(0, T_{\varepsilon}\right) \text {, }
$$


where $T_{\varepsilon}:=\min \left\{1, \frac{1}{2} T_{\max , \varepsilon}\right\}$. Then given $\tau_{0}>0$, for any such $\varepsilon$ we can fix $\tau=\tau\left(\varepsilon, \tau_{0}\right)>0$ such that $\tau \leq \tau_{0}$ and $\tau<T_{\varepsilon}$ as well as

$$
\tau \leq \frac{1}{(\mu-\chi) c_{1}(\varepsilon)}
$$

because $\mu>\chi$. Introducing

$$
\bar{u}(x, t):=\frac{1}{(\mu-\chi) t}+\frac{\kappa_{+}}{\mu-\chi}, \quad x \in \bar{\Omega}, \quad t \geq \tau,
$$

from (2.10) and (2.11) we therefore obtain that

$$
\bar{u}(x, \tau) \geq \frac{1}{(\mu-\chi) \tau} \geq c_{1}(\varepsilon) \geq u_{\varepsilon}(x, \tau) \quad \text { for all } x \in \Omega,
$$

and clearly $\frac{\partial \bar{u}(x, t)}{\partial v}=0=\frac{\partial u_{\varepsilon}(x, t)}{\partial v}$ for all $x \in \partial \Omega$ and $t \in\left(\tau, T_{\max , \varepsilon}\right)$. Furthermore, differentiating in (2.12) we see that

$$
\begin{aligned}
\bar{u}_{t} & -\Delta \bar{u}+\chi \nabla v_{\varepsilon} \cdot \nabla \bar{u}+\chi m_{\varepsilon}(t) \bar{u}-\kappa \bar{u}+(\mu-\chi) \bar{u}^{2} \\
& =\bar{u}_{t}+\chi m_{\varepsilon}(t) \bar{u}-\kappa \bar{u}+(\mu-\chi) \bar{u}^{2} \\
& \geq \bar{u}_{t}-\kappa+\bar{u}+(\mu-\chi) \bar{u}^{2} \\
& =-\frac{1}{(\mu-\chi) t^{2}}-\kappa_{+} \cdot\left\{\frac{1}{(\mu-\chi) t}+\frac{\kappa_{+}}{\mu-\chi}\right\}+(\mu-\chi) \cdot\left\{\frac{1}{(\mu-\chi) t}+\frac{\kappa_{+}}{\mu-\chi}\right\}^{2} \\
& =\frac{\kappa_{+}}{(\mu-\chi) t} \\
& \geq 0 \text { for all } x \in \Omega \text { and } t \in\left(\tau, T_{\max , \varepsilon}\right),
\end{aligned}
$$

whence in view of (2.8) the comparison principle applies so as to assert the inequality $\bar{u} \geq u_{\varepsilon}$ throughout $\bar{\Omega} \times\left[\tau, T_{\max , \varepsilon}\right) \supset \bar{\Omega} \times\left[\tau_{0}, T_{\max , \varepsilon}\right)$. Firstly fixing, e.g., $\tau_{0}:=T_{\varepsilon}$ here, from the extensibility criterion in Lemma 2.1 we thereby infer that indeed $T_{\max , \varepsilon}=\infty$ for all $\varepsilon \in(0,1)$, whereupon we secondly achieve (2.9) upon taking $\tau_{0} \searrow 0$.

\subsection{Downward radial monotonicity of both solution components}

Besides the mere radial symmetry, a further indispensable prerequisite for the most essential among our subsequent arguments will be provided by the observation that the initially assumed monotonicity property expressed in (1.4) and (2.2) is inherited by $u_{\varepsilon}$ and hence in fact also carries over to $v_{\varepsilon}$. Both these statements rely on the following elementary fact.

Lemma 2.3 Let $\kappa \in \mathbb{R}$ and $0<\chi<\mu$, and assume (1.4). Then for all $\varepsilon \in(0,1)$, we have

$$
r^{n-1} v_{\varepsilon r}(r, t)=\frac{1}{n} m_{\varepsilon}(t) r^{n}-\int_{0}^{r} \rho^{n-1} u_{\varepsilon}(\rho, t) \mathrm{d} \rho \quad \text { for all } r \in(0, R) \text { and } t>0 .
$$

Proof Since when written in radial variables the second equation in (2.6) becomes

$$
\left(r^{n-1} v_{\varepsilon r}\right)_{r}=r^{n-1} m_{\varepsilon}(t)-r^{n-1} u_{\varepsilon}(r, t) \quad \text { for all } r \in(0, R) \text { and } t>0,
$$

the claim directly results upon integration over $(0, r)$ for $r \in(0, R)$.

Indeed, this enables us to suitably reduce the differentiated version of (2.8) so as to conclude, again by comparison and again making use of the assumption $\mu>\chi$, that $u_{\varepsilon}$ remains radially nonincreasing. 
Lemma 2.4 Assume that $\kappa \in \mathbb{R}$ and $0<\chi<\mu$, and that (1.4) holds. Then for each $\varepsilon \in(0,1)$,

$$
u_{\varepsilon r}(r, t) \leq 0 \quad \text { for all } r \in(0, R) \text { and } t>0 .
$$

Proof We fix $\varepsilon \in(0,1)$ and $T>0$ and then choose $\lambda_{\varepsilon}>0$ large enough fulfilling

$$
\lambda_{\varepsilon}>\kappa_{+}+3 \chi\left\|u_{\varepsilon}\right\|_{L^{\infty}(\Omega \times(0, T))},
$$

and for $\eta>0$ we let

$$
z_{\varepsilon \eta}(r, t):=u_{\varepsilon r}(r, t)-\eta e^{\lambda_{\varepsilon} t}, \quad r \in[0, R], \quad t \in[0, T] .
$$

Then since due to (2.1) and (2.3) classical parabolic theory [19] ensures that $u_{\varepsilon r} \in C^{0}([0, R] \times$ $[0, T]) \cap C^{2,1}([0, R] \times(0, T])$ and that $u_{\varepsilon r}(0, t)=u_{\varepsilon r}(R, t)=0$ for all $t \in[0, T]$, it follows that $z_{\varepsilon \eta}$ belongs to the same space and satisfies

$$
z_{\varepsilon \eta}(0, t)=z_{\varepsilon \eta}(R, t)=-\eta e^{\lambda_{\varepsilon} t}<0 \quad \text { for all } t \in[0, T]
$$

as well as

$$
z_{\varepsilon \eta}(r, 0)=u_{0 \varepsilon r}(r)-\eta \leq-\eta<0 \quad \text { for all } r \in[0, R]
$$

according to (2.2). Moreover, differentiating the identity (2.8) with respect to $r=|x|$, we see that

$$
u_{\varepsilon r t}=u_{\varepsilon r r r}+a_{\varepsilon}(r, t) u_{\varepsilon r r}+b_{\varepsilon}(r, t) u_{\varepsilon r} \quad \text { for all } r \in(0, R) \text { and } t \in(0, T),
$$

with

$$
a_{\varepsilon}(r, t):=\frac{n-1}{r}-\chi v_{\varepsilon r}, \quad r \in(0, R), \quad t \in(0, T),
$$

and

$$
b_{\varepsilon}(r, t):=-\frac{n-1}{r^{2}}-\chi v_{\varepsilon r r}-\chi m_{\varepsilon}(t)+\kappa-2(\mu-\chi) u_{\varepsilon}, \quad r \in(0, R), \quad t \in(0, T),
$$

and that hence

$$
\begin{aligned}
z_{\varepsilon \eta t}= & z_{\varepsilon \eta r r}+a_{\varepsilon}(r, t) z_{\varepsilon \eta r}+b_{\varepsilon}(r, t) z_{\varepsilon \eta}+\left(b_{\varepsilon}(r, t)-\lambda_{\varepsilon}\right) \cdot \eta e^{\lambda_{\varepsilon} t} \\
& \text { for all } r \in(0, R) \text { and } t \in(0, T) .
\end{aligned}
$$

Here from the second equation in (2.6), we know that

$$
v_{\varepsilon r r}=m_{\varepsilon}(t)-u_{\varepsilon}-\frac{n-1}{r} v_{\varepsilon r} \quad \text { for all } r \in(0, R) \text { and } t \in(0, T),
$$

and that, by Lemma 2.3 ,

$$
\begin{aligned}
v_{\varepsilon r}(r, t)= & \frac{1}{n} m_{\varepsilon}(t) r-r^{1-n} \int_{0}^{r} \rho^{n-1} u_{\varepsilon}(\rho, t) \mathrm{d} \rho \leq \frac{1}{n} m_{\varepsilon}(t) r \\
& \text { for all } r \in(0, R) \text { and } t \in(0, T),
\end{aligned}
$$

whence

$$
-\chi v_{\varepsilon r r} \leq-\chi m_{\varepsilon}(t)+\chi u_{\varepsilon}+\frac{(n-1) \chi}{r} \cdot \frac{1}{n} m_{\varepsilon}(t) r=-\frac{\chi}{n} m_{\varepsilon}(t)+\chi u_{\varepsilon} \leq \chi u_{\varepsilon}
$$

for all $r \in(0, R)$ and $t \in(0, T)$. Therefore, (2.15) ensures that

$$
b_{\varepsilon}(r, t) \leq \chi u_{\varepsilon}-\chi m_{\varepsilon}(t)+\kappa-2(\mu-\chi) u_{\varepsilon} \leq \kappa_{+}+3 \chi u_{\varepsilon} \leq \lambda_{\varepsilon}
$$


for all $r \in(0, R)$ and $t \in(0, T)$,

and that thus

$$
z_{\varepsilon \eta t} \leq z_{\varepsilon \eta r r}+a_{\varepsilon}(r, t) z_{\varepsilon \eta r}+b_{\varepsilon}(r, t) z_{\varepsilon \eta} \quad \text { for all } r \in(0, R) \text { and } t \in(0, T)
$$

according to (2.18). As a consequence of (2.16) and (2.17), it thus follows from a standard maximum principle argument that in none of the domains $[0, R] \times\left[0, T_{0}\right], T_{0} \in(0, T], z_{\varepsilon \eta}$ can attain its maximal value 0 , and that hence $z_{\varepsilon \eta}$ actually must remain negative throughout $[0, R] \times[0, T]$, which on taking $\eta \searrow 0$ implies (2.14).

Fortunately, the structure of the equation in (2.6) governing $v_{\varepsilon}$, actually reducing to (2.13), is simple enough so as to allow for a similar conclusion concerning the second solution component:

Lemma 2.5 If $\kappa \in \mathbb{R}, 0<\chi<\mu$ and (1.4) holds, then for arbitrary $\varepsilon \in(0,1)$,

$$
v_{\varepsilon r}(r, t) \leq 0 \quad \text { for all } r \in(0, R) \text { and } t>0 .
$$

Proof We fix $t>0$ and then obtain from the downward monotonicity of $[0, R] \ni r \mapsto$ $u_{\varepsilon}(r, t)$, as asserted by Lemma 2.4 , that for all $r \in(0, R)$,

$$
\begin{aligned}
\frac{n}{r^{n}} \int_{0}^{r} \rho^{n-1} u_{\varepsilon}(\rho, t) \mathrm{d} \rho & =\frac{1}{\left|B_{r}(0)\right|} \int_{B_{r}(0)} u_{\varepsilon}(x, t) \mathrm{d} x \\
& \geq \frac{1}{\left|B_{R}(0)\right|} \int_{B_{R}(0)} u_{\varepsilon}(x, t) \mathrm{d} x \\
& =m_{\varepsilon}(t) .
\end{aligned}
$$

In view of (2.13), this precisely means that $v_{\varepsilon r}(r, t) \leq 0$ for all $r \in(0, R)$.

\subsection{Constructing a smooth limit in $\times(0, \infty)$}

Returning to the outcome of Lemma 2.2, we can now proceed to the construction of a radially decreasing limit couple $(u, v)$ which is smooth for $t>0$ and solves the corresponding identities in (1.2) classically in this region. Indeed, by quite a straightforward reasoning we obtain the following result on higher regularity away from the temporal origin.

Lemma 2.6 Let $\kappa \in \mathbb{R}$ and $0<\chi<\mu$, and assume (1.4). Then for all $\tau \in(0,1)$ and $T>1$ there exist $\theta=\theta(\tau, T) \in(0,1)$ and $C(\tau, T)>0$ such that

$$
\left\|u_{\varepsilon}\right\|_{C^{2+\theta, 1+\frac{\theta}{2}}(\bar{\Omega} \times[\tau, T])}+\left\|v_{\varepsilon}\right\|_{C^{2+\theta, \frac{\theta}{2}}(\bar{\Omega} \times[\tau, T])} \leq C(\tau, T) \quad \text { for all } \varepsilon \in(0,1) .
$$

Proof On the basis of the uniform bound for $u_{\varepsilon}$ in $L^{\infty}(\Omega \times(\tau, \infty))$ provided by Lemma 2.2 for each $\tau \in(0,1)$, this can be seen by adapting a standard bootstrap procedure to the present setting in a straightforward manner (cf., e.g., [10, Section 5]).

The compactness features thereby provided directly entail the announced existence result.

Lemma 2.7 Assume that $\kappa \in \mathbb{R}$ and $0<\chi<\mu$, and that (1.4) holds. Then there exists $\left(\varepsilon_{j}\right)_{j \in \mathbb{N}} \subset(0,1)$ such that $\varepsilon_{j} \searrow 0$ as $j \rightarrow \infty$, and such that as $\varepsilon=\varepsilon_{j} \searrow 0$ we have

$$
u_{\varepsilon} \rightarrow u \text { in } C_{\mathrm{loc}}^{2,1}(\bar{\Omega} \times(0, \infty))
$$


and

$$
v_{\varepsilon} \rightarrow v \text { in } C_{\text {loc }}^{2,0}(\bar{\Omega} \times(0, \infty))
$$

with some nonnegative functions $u$ and $v$ which form a classical solution of the boundary value problem in $(1.2)$ in $\bar{\Omega} \times(0, \infty)$. Moreover, $u(\cdot, t)$ and $v(\cdot, t)$ are radially symmetric and nonincreasing with respect to $|x|$ for all $t>0$.

Proof According to Lemma 2.6, this can readily be derived by using the Arzelà-Ascoli theorem and an appropriate limit passage in (2.6), Lemmas 2.4 and 2.5.

\section{Linking regularity at $t=0$ to time integrability of total mass}

We next approach the core of our analysis by focusing on the initial behavior of the solution $(u, v)$ obtained in Lemma 2.7, and it will turn out that a crucial role in this regard is played by the behavior of the total mass functional $\int_{\Omega} u(x, t) \mathrm{d} x$ near $t=0$, which through the nonlocal coupling expressed in (2.13) is closely linked to regularity of the cross-diffusive gradient $v_{\varepsilon r}$. Here bearing in mind our ultimate goal of treating initial data merely fulfilling, e.g., (1.14), we may by far not expect boundedness of this functional, while on the other hand the bound therefore trivially implied by the upper estimate for $u_{\varepsilon}$ from Lemma 2.2 appears to be too rough by only yielding a multiple of $\frac{1}{t}$ for $t<1$ as a majorant not integrable near $t=0$. Driven by the observation that, as we shall see by an independent reasoning in Sect. 3.1, such an integrability feature seems essential for any nontrivial solution behavior near $t=0$, in Sect. 3.2 we will employ another comparison argument to derive a further pointwise upper bound for $u_{\varepsilon}$ which is now singular at the spatial origin but uniform in time. In Sect. 3.3 this will be seen to imply an integrable control for the mass functional, which will thereafter entail attainment of the initial trace firstly with respect to some quite rough topology (Sect. 3.4), and finally, upon another spatially local regularity reasoning in Sect. 3.5, also in the desired locally uniform sense (Sect. 3.6).

\subsection{Necessity of temporal mass integrability for nontrivial initial traces}

Our further considerations can be motivated by the following observation indicating a crucial role that integrability of the total mass functional plays with regard to nontrivial attainment of initial traces.

Proposition 3.1 Let $\kappa \in \mathbb{R}$ and $0<\chi<\mu$, and suppose that for some $T>0,(u, v) \in$ $C^{2,1}(\bar{\Omega} \times(0, T]) \times C^{2,0}(\bar{\Omega} \times(0, T])$ is a classical solution of the boundary value problem in $(1.2)$ in $\bar{\Omega} \times(0, T]$ which is such that $u(\cdot, t)$ and $v(\cdot, t)$ are radially symmetric and nonincreasing with respect to $|x| \in(0, R)$ for all $t \in(0, T)$, and such that

$$
\int_{0}^{\mathrm{T}} \int_{\Omega} u(x, t) \mathrm{d} x \mathrm{~d} t=\infty .
$$

If, apart from that,

$$
\liminf _{t \searrow 0} u(x, t)<\infty \quad \text { for some } x \in \Omega,
$$

then for all $r_{0} \in(|x|, R)$ we have

$$
\liminf _{t \searrow 0}\|u(\cdot, t)\|_{L^{\infty}\left(\Omega \backslash B_{r_{0}}(0)\right)}=0 .
$$


In particular, if there exists $\widehat{u}_{0}: \Omega \rightarrow \mathbb{R}$ such that

$$
u(\cdot, t) \rightarrow \widehat{u}_{0} \quad \text { a.e. in } \Omega \quad \text { as } t \searrow 0,
$$

then

$$
\widehat{u}_{0}=0 \quad \text { a.e. in } \Omega \text {. }
$$

Proof Fixing $x \in \Omega$ such that (3.2) holds, this hypothesis states the existence of $c_{1}>0$ and $\left(t_{k}\right)_{k \in \mathbb{N}} \subset(0, T)$ such that $t_{k} \searrow 0$ as $k \rightarrow \infty$, and such that writing $r_{\star}:=|x| \in[0, R)$ and again using radial variables we have $u\left(r_{\star}, t_{k}\right) \leq c_{1}$ for all $k \in \mathbb{N}$. As also by assumption we know that $u_{r}\left(\cdot, t_{k}\right) \leq 0$ in $(0, R)$, this in particular entails that

$$
y(t):=\int_{r^{\star}}^{R}\left(r-r_{\star}\right)^{4} u(r, t) \mathrm{d} r, \quad t \in(0, T),
$$

satisfies

$$
y\left(t_{k}\right) \leq c_{2}:=c_{1} \int_{r_{\star}}^{R}\left(r-r_{\star}\right)^{4} \mathrm{~d} r=\frac{c_{1}\left(R-r_{\star}\right)^{5}}{5} \quad \text { for all } k \in \mathbb{N} .
$$

Now if (3.3) was false for some $r_{0} \in\left(r_{\star}, R\right)$, then we could find $c_{3}>0$ and $\tau \in(0, T)$ with the property that $u\left(r_{0}, t\right) \geq c_{3}$ for all $t \in(0, \tau)$, again by monotonicity of $(0, R) \ni r \mapsto u(r, t)$ meaning that

$$
y(t) \geq c_{4}:=c_{3} \cdot \int_{r_{\star}}^{r_{0}}\left(r-r_{\star}\right)^{4} \mathrm{~d} r=\frac{c_{3}\left(r_{0}-r_{\star}\right)^{5}}{5} \quad \text { for all } t \in(0, \tau) .
$$

To derive a contradiction from this, in full analogy to (2.8), we combine the first two equations in (1.2) to see that

$u_{t}=u_{r r}+\frac{n-1}{r} u_{r}-\chi u_{r} v_{r}-\chi m(t) u+\kappa u-(\mu-\chi) u^{2} \quad$ for $r \in(0, R)$ and $t \in(0, T)$,

with $m(t)=\frac{n}{R^{n}} \int_{0}^{R} r^{n-1} u(r, t) \mathrm{d} r, t \in(0, T)$, fulfilling

$$
\int_{0}^{\tau} m(t) \mathrm{d} t=\infty
$$

as a consequence of (3.1). Moreover, since our assumptions ensure that $u_{r}$ and $v_{r}$ are nonnegative, (3.9) implies that

$$
u_{t} \leq u_{r r}-\chi m(t) u+\kappa u-(\mu-\chi) u^{2} \quad \text { in }(0, R) \times(0, T),
$$

and that thus the function $y$ from (3.6) satisfies

$$
\begin{aligned}
y^{\prime}(t) \leq & \int_{r_{\star}}^{R}\left(r-r_{\star}\right)^{4} u_{r r}(r, t) \mathrm{d} r-\chi m(t) y(t)+\kappa y(t) \\
& -(\mu-\chi) \int_{r_{\star}}^{R}\left(r-r_{\star}\right)^{4} u^{2}(r, t) \mathrm{d} r \quad \text { for all } t \in(0, T) .
\end{aligned}
$$

Here two integrations by parts show that since $u_{r}(R, t)=0$ for all $t \in(0, T)$,

$$
\begin{aligned}
\int_{r_{\star}}^{R}\left(r-r_{\star}\right)^{4} u_{r r}(r, t) \mathrm{d} r & =12 \int_{r_{\star}}^{R}\left(r-r_{\star}\right)^{2} u(r, t) \mathrm{d} r \\
& +\left[\left(r-r_{\star}\right)^{4} u_{r}(r, t)-4\left(r-r_{\star}\right)^{3} u(r, t)\right]_{r=r_{\star}}^{r=R}
\end{aligned}
$$




$$
\begin{aligned}
& =12 \int_{r_{\star}}^{R}\left(r-r_{\star}\right)^{2} u(r, t) \mathrm{d} r-4\left(R-r_{\star}\right)^{3} u(R, t) \\
& \leq 12 \int_{r_{\star}}^{R}\left(r-r_{\star}\right)^{2} u(r, t) \mathrm{d} r \quad \text { for all } t \in(0, T),
\end{aligned}
$$

so that using Young's inequality along with our assumption that $\mu>\chi$ we obtain that

$$
\int_{r_{\star}}^{R}\left(r-r_{\star}\right)^{4} u_{r r}(r, t) \mathrm{d} r \leq \frac{\mu-\chi}{2} \int_{r_{\star}}^{R}\left(r-r_{\star}\right)^{4} u^{2}(r, t) \mathrm{d} r+c_{5} \quad \text { for all } t \in(0, T)
$$

with $c_{5}:=\frac{72\left(R-r_{\star}\right)}{\mu-\chi}>0$. As, by the same token, writing $c_{6}:=\frac{\kappa_{+}^{2}}{2(\mu-\chi)} \int_{r_{\star}}^{R}\left(r-r_{\star}\right)^{4} \mathrm{~d} r=$ $\frac{\kappa_{+}^{2}\left(r-r_{\star}\right)^{5}}{10(\mu-\chi)}$, we have

$$
\kappa y(t) \leq \frac{\mu-\chi}{2} \int_{r_{\star}}^{R}\left(r-r_{\star}\right)^{4} u^{2}(r, t) \mathrm{d} r+c_{6} \quad \text { for all } t \in(0, T),
$$

from (3.11) we infer that

$$
y^{\prime}(t)+\chi m(t) y(t) \leq c_{5}+c_{6} \quad \text { for all } t \in(0, T),
$$

and that hence, after integration,

$$
y(\tau)+\chi \int_{t_{k}}^{\tau} m(t) y(t) \mathrm{d} t \leq y\left(t_{k}\right)+\left(c_{5}+c_{6}\right) \cdot\left(\tau-t_{k}\right) \quad \text { for all } k \geq k_{0}
$$

if we let $k_{0} \in \mathbb{N}$ be large enough such that $t_{k}<\tau$ for all $k \geq k_{0}$. In view of (3.8) and (3.7), however, this particularly entails that

$$
c_{4} \chi \int_{t_{k}}^{\tau} m(t) \mathrm{d} t \leq c_{2}+\left(c_{5}+c_{6}\right) \tau \quad \text { for all } k \geq k_{0},
$$

which, due to the positivity of $\chi$, in the limit $k \rightarrow \infty$ contradicts (3.10) and thereby completes the proof.

\subsection{A spatially singular but temporally uniform pointwise upper bound for $u_{\varepsilon}$}

Relying on the fact that (1.7) guarantees a favorable supersolution property enjoyed by large multiples of $\phi$ for solutions of (2.8) with nonincreasing derivatives $u_{\varepsilon r}$ and $v_{\varepsilon r}$, we can turn our hypothesis (1.5) into a second pointwise bound for $u_{\varepsilon}$ of the announced flavor.

Lemma 3.2 Assume that $\kappa \in \mathbb{R}$ and $0<\chi<\mu$, and suppose that beyond (1.4), $u_{0}$ satisfies (1.5) with some $K>0$ and some $\phi$ fulfilling (1.6) and (1.7) with a certain $K_{1}>0$. Then there exists $C>0$ such that for each $\varepsilon \in(0,1)$,

$$
u_{\varepsilon}(r, t) \leq C \phi(r) \quad \text { for all } r \in(0, R) \text { and } t>0 .
$$

Proof Using that $\mu>\chi$ and that $\phi(R)$ is positive, we choose a positive constant $c_{1}$ large enough such that

$$
c_{1} \geq \frac{2 K_{1}}{\mu-\chi}
$$

and

$$
c_{1} \geq \frac{2 \kappa_{+}}{(\mu-\chi) \phi(R)}
$$


as well as

$$
c_{1} \geq K+\frac{1}{\phi(R)} .
$$

For fixed $\varepsilon \in(0,1)$ and $\delta_{0} \in(0, R)$ we may then rely on the boundedness of $u_{\varepsilon}$ in $\Omega \times(0, \infty)$, as asserted by Lemma 2.1 when combined with Lemma 2.2, to find $c_{2}(\varepsilon)>0$ such that

$$
u_{\varepsilon}(r, t) \leq c_{2}(\varepsilon) \quad \text { for all } r \in(0, R) \text { and } t>0,
$$

and recall that by (1.6) we have $\phi(r) \nearrow+\infty$ as $r \searrow 0$, so that we can pick $\delta=\delta\left(\varepsilon, \delta_{0}\right) \in$ $(0, R)$ such that $\delta \leq \delta_{0}$ and

$$
\phi(\delta) \geq \frac{c_{2}(\varepsilon)}{c_{1}} .
$$

Here the latter together with (3.16) ensures that

$$
\bar{u}(r, t):=c_{1} \phi(r), \quad r \in[0, R], \quad t \geq 0,
$$

satisfies

$$
\bar{u}(\delta, t)=c_{1} \phi(\delta) \geq c_{2}(\varepsilon) \geq u_{\varepsilon}(\delta, t) \quad \text { for all } t>0 .
$$

Moreover, by monotonicity of $\phi$,

$$
\bar{u}_{r}(R, t)=c_{1} \phi^{\prime}(R) \leq 0=u_{\varepsilon r}(R, t) \quad \text { for all } t>0,
$$

and at the initial time, our assumption (1.5) along with (2.4) warrants that due to (3.15) we have

$$
\begin{aligned}
\bar{u}(r, 0) & =K \phi(r)+\left(c_{1}-K\right) \phi(r) \\
& \geq K \phi(r)+\left(c_{1}-K\right) \phi(R) \\
& \geq u_{0}(r)+1 \\
& \geq u_{0 \varepsilon}(r) \quad \text { for all } r \in(\delta, R) .
\end{aligned}
$$

Now computing

$$
\begin{gathered}
\bar{u}_{t}-\bar{u}_{r r}-\frac{n-1}{r} \bar{u}_{r}-\kappa \bar{u}+(\mu-\chi) \bar{u}^{2}=c_{1} \phi^{\prime \prime}(r)-c_{1} \cdot \frac{n-1}{r} \phi^{\prime}(r) \\
-c_{1} \kappa \phi(r)+c_{1}^{2}(\mu-\chi) \phi^{2}(r), \quad r \in(\delta, R), \quad t>0,
\end{gathered}
$$

we see that again due to the monotonicity of $\phi$, by using (3.14) we may estimate

$$
-c_{1} \cdot \frac{n-1}{r} \phi^{\prime}(r) \geq 0 \quad \text { for all } r \in(\delta, R)
$$

and

$$
\frac{c_{1} \kappa \phi(r)}{\frac{1}{2} c_{1}^{2}(\mu-\chi) \phi^{2}(r)}=\frac{2 \kappa}{c_{1}(\mu-\chi) \phi(r)} \leq \frac{2 \kappa_{+}}{c_{1}(\mu-\chi) \phi(R)} \leq 1 \quad \text { for all } r \in(\delta, R) .
$$

As finally (1.7) in view of (3.13) implies that also

$$
\frac{c_{1} \phi^{\prime \prime}(r)}{\frac{1}{2} c_{1}^{2}(\mu-\chi) \phi^{2}(r)}=\frac{2 \phi^{\prime \prime}(r)}{(\mu-\chi) c_{1} \phi^{2}(r)} \leq \frac{2 K_{1}}{(\mu-\chi) c_{1}} \leq 1 \quad \text { for all } r \in(\delta, R),
$$

from (3.21) it follows that

$$
\bar{u}_{t} \geq \bar{u}_{r r}+\frac{n-1}{r} \bar{u}_{r}+\kappa \bar{u}-(\mu-\chi) \bar{u}^{2} \quad \text { for all } r \in(\delta, R) \text { and any } t>0,
$$


while thanks to the inequalities $u_{\varepsilon r} \leq 0$ and $v_{\varepsilon r} \leq 0$ asserted by Lemmas 2.4 and 2.5, respectively, from (2.8) we obtain that

$$
\begin{aligned}
u_{\varepsilon t} & =u_{\varepsilon r r}+\frac{n-1}{r} u_{\varepsilon r}-\chi u_{\varepsilon r} v_{\varepsilon r}-\chi m_{\varepsilon}(t) u_{\varepsilon}+\kappa u_{\varepsilon}-(\mu-\chi) u_{\varepsilon}^{2} \\
& \leq u_{\varepsilon r r}+\frac{n-1}{r} u_{\varepsilon r}+\kappa u_{\varepsilon}-(\mu-\chi) u_{\varepsilon}^{2} \quad \text { for all } r \in(\delta, R) \text { and } t>0 .
\end{aligned}
$$

On the basis of (3.18)-(3.20), we may therefore conclude by a comparison argument that $\bar{u}(r, t) \geq u_{\varepsilon}(r, t)$ for all $r \in\left(\delta\left(\varepsilon, \delta_{0}\right), R\right)$ and $t>0$, which since $\delta\left(\varepsilon, \delta_{0}\right) \leq \delta_{0}$ entails that

$$
u_{\varepsilon}(r, t) \leq c_{1} \phi(r) \quad \text { for all } r \in\left(\delta_{0}, R\right) \text { and } t>0
$$

and therefore establishes (3.12) in the limit $\delta_{0} \searrow 0$.

\subsection{Uniform integrability of the mass functional}

Now having at hand the upper inequalities for $u_{\varepsilon}$ both from Lemma 2.2 and from Lemma 3.2, on combining these and relying on the technical assumption (1.8), we can construct a majorant for the mass functional in the following sense.

Lemma 3.3 Let $\kappa \in \mathbb{R}$ and $0<\chi<\mu$, and assume that (1.4) and (1.5) hold with some $K>0$ and some $\phi$ satisfying (1.6) and (1.7) as well as (1.8) with some $K_{1}>0$ and $K_{2}>0$. Then there exist $t_{0} \in(0,1)$ and $C>0$ such that for any $\varepsilon \in(0,1)$ we have

$$
\int_{\Omega} u_{\varepsilon}(x, t) \mathrm{d} x \leq C \cdot \frac{\left[\phi^{-1}\left(\frac{1}{t}\right)\right]^{n}}{t} \quad \text { for all } t \in\left(0, t_{0}\right) .
$$

Proof According to Lemma 2.2, we can find $c_{1}>0$ such that for all $\varepsilon \in(0,1)$,

$$
u_{\varepsilon}(r, t) \leq \frac{c_{1}}{t} \quad \text { for all } r \in(0, R) \text { and each } t \in(0,1),
$$

while Lemma 3.2 provides $c_{2}>0$ fulfilling

$$
u_{\varepsilon}(r, t) \leq c_{2} \phi(r) \quad \text { for all } r \in(0, R) \text { and } t>0
$$

whenever $\varepsilon \in(0,1)$. Thus, if we let $t_{0} \in(0,1)$ be such that $t_{0} \leq \frac{1}{\phi\left(\frac{R}{2}\right)}$, then for $t \in\left(0, t_{0}\right)$ we have $2 \phi^{-1}\left(\frac{1}{t}\right)<2 \phi^{-1}\left(\phi\left(\frac{R}{2}\right)\right)=R$, and hence splitting

$$
\int_{0}^{R} r^{n-1} u_{\varepsilon}(r, t) \mathrm{d} r=\int_{0}^{2 \phi^{-1}\left(\frac{1}{t}\right)} r^{n-1} u_{\varepsilon}(r, t) \mathrm{d} r+\int_{2 \phi^{-1}\left(\frac{1}{t}\right)}^{R} r^{n-1} u_{\varepsilon}(r, t) \mathrm{d} r,
$$

we can combine (3.23) with (3.24) to estimate

$$
\begin{aligned}
\int_{0}^{2 \phi^{-1}\left(\frac{1}{t}\right)} r^{n-1} u_{\varepsilon}(r, t) \mathrm{d} r & \leq \frac{c_{1}}{t} \cdot \int_{0}^{2 \phi^{-1}\left(\frac{1}{t}\right)} r^{n-1} \mathrm{~d} r \\
& =\frac{2^{n} c_{1}}{n} \cdot \frac{\left[\phi^{-1}\left(\frac{1}{t}\right)\right]^{n}}{t}
\end{aligned}
$$

and

$$
\int_{2 \phi^{-1}\left(\frac{1}{t}\right)}^{R} r^{n-1} u_{\varepsilon}(r, t) \mathrm{d} r \leq c_{2} \phi\left(2 \phi^{-1}\left(\frac{1}{t}\right)\right) \cdot \int_{2 \phi^{-1}\left(\frac{1}{t}\right)}^{R} r^{n-1} \mathrm{~d} r
$$




$$
\leq \frac{c_{2} R^{n}}{n} \cdot \phi\left(2 \phi^{-1}\left(\frac{1}{t}\right)\right)
$$

for all $t \in\left(0, t_{0}\right)$. Now since (1.8) ensures that herein

$$
\begin{aligned}
\phi\left(2 \phi^{-1}\left(\frac{1}{t}\right)\right) & \leq K_{2} \cdot\left[2 \phi^{-1}\left(\frac{1}{t}\right)\right]^{n} \cdot \phi\left(\phi^{-1}\left(\frac{1}{t}\right)\right) \\
& =2^{n} K_{2} \cdot \frac{\left[\phi^{-1}\left(\frac{1}{t}\right)\right]^{n}}{t} \quad \text { for all } t \in(0,1),
\end{aligned}
$$

from (3.25) it follows that if we abbreviate $c_{3}:=\frac{2^{n} c_{1}}{n}+\frac{c_{2} R^{n}}{n} \cdot 2^{n} K_{2}$, then

$$
\int_{0}^{R} r^{n-1} u_{\varepsilon}(r, t) \mathrm{d} r \leq c_{2} \cdot \frac{\left[\phi^{-1}\left(\frac{1}{t}\right)\right]^{n}}{t} \quad \text { for all } t \in\left(0, t_{0}\right),
$$

which precisely yields (3.22).

Now our final and most crucial hypothesis (1.9) ensures integrability of the right-hand side in (3.22) near $t=0$ :

Lemma 3.4 Suppose that $\phi$ satisfies (1.6) as well as (1.9). Then

$$
\int_{0}^{t_{0}} \frac{\left[\phi^{-1}\left(\frac{1}{t}\right)\right]^{n}}{t} \mathrm{~d} t<\infty \quad \text { for all } t_{0} \in(0,1) .
$$

Proof Substituting $r=\phi^{-1}\left(\frac{1}{t}\right)$ and integrating by parts, for $\delta \in\left(0, t_{0}\right)$ we can rewrite

$$
\begin{aligned}
\int_{\delta}^{t_{0}} \frac{\left[\phi^{-1}\left(\frac{1}{t}\right)\right]^{n}}{t} \mathrm{~d} t= & -\int_{\phi^{-1}\left(\frac{1}{\delta}\right)}^{\phi^{-1}\left(\frac{1}{t_{0}}\right)} \frac{r^{n}}{\frac{1}{\phi(r)}} \cdot \frac{\phi^{\prime}(r)}{\phi^{2}(r)} \mathrm{d} r \\
= & -\int_{\phi^{-1}\left(\frac{1}{\delta}\right)}^{\phi^{-1}\left(\frac{1}{t_{0}}\right)} r^{n} \cdot \frac{d}{\mathrm{~d} r} \ln \phi(r) \mathrm{d} r \\
= & n \int_{\phi^{-1}\left(\frac{1}{\delta}\right)}^{\phi^{-1}\left(\frac{1}{t_{0}}\right)} r^{n-1} \ln \phi(r) \mathrm{d} r \\
& -\left[\phi^{-1}\left(\frac{1}{t_{0}}\right)\right]^{n} \cdot \ln \frac{1}{t_{0}}+\left[\phi^{-1}\left(\frac{1}{\delta}\right)\right]^{n} \cdot \ln \frac{1}{\delta}
\end{aligned}
$$

Here, clearly,

$$
\begin{aligned}
\int_{\phi^{-1}\left(\frac{1}{\delta}\right)}^{\phi^{-1}\left(\frac{1}{t_{0}}\right)} r^{n-1} \ln \phi(r) \mathrm{d} r \leq & n \int_{0}^{1} r^{n-1} \ln \phi(r) \mathrm{d} r+n \int_{1}^{\phi^{-1}\left(\frac{1}{t_{0}}\right)} r^{n-1} \ln \phi(1) \mathrm{d} r \\
\leq & c_{1}:=n \int_{0}^{1} r^{n-1} \ln \phi(r) \mathrm{d} r+\left[\phi^{-1}\left(\frac{1}{t_{0}}\right)\right]^{n} \cdot \ln \phi(1) \\
& \quad \text { for all } \delta \in\left(0, t_{0}\right),
\end{aligned}
$$

with $c_{1}$ being finite and positive by (1.9) and (1.6). Apart from that, (1.9) ensures that there must exist $\left(r_{j}\right)_{j \in \mathbb{N}} \subset\left(0, \phi^{-1}\left(\frac{1}{t_{0}}\right)\right)$ such that $r_{j} \searrow 0$ as $j \rightarrow \infty$ and $r_{j}^{n-1} \ln \phi\left(r_{j}\right) \leq \frac{1}{r_{j}}$ for all $j \in \mathbb{N}$, for otherwise $\int_{0}^{1} r^{n-1} \ln \phi(r) \mathrm{d} r \geq \int_{0}^{1} \frac{\mathrm{d} r}{r}=\infty$. The numbers $\delta_{j}:=\frac{1}{\phi\left(r_{j}\right)}, j \in \mathbb{N}$ thus satisfy $\delta_{j}<t_{0}$ as well as

$$
\left[\phi^{-1}\left(\frac{1}{\delta_{j}}\right)\right]^{n} \cdot \ln \frac{1}{\delta_{j}}=r_{j}^{n} \ln \phi\left(r_{j}\right) \leq 1
$$


for all $j \in \mathbb{N}$, whence (3.27) along with (3.28) entails that

$$
\int_{\delta_{j}}^{t_{0}} \frac{\left[\phi^{-1}\left(\frac{1}{t}\right)\right]^{n}}{t} \mathrm{~d} t \leq c_{1}+1 \quad \text { for all } j \in \mathbb{N},
$$

because $\ln \frac{1}{t_{0}}$ is positive. Since the assumed convergence property of $\left(r_{j}\right)_{j \in \mathbb{N}}$ together with (1.6) warrants that $\delta_{j} \searrow 0$ as $j \rightarrow \infty$, this establishes (3.26).

A further and quite immediate consequence of Lemma 3.3 on $v_{\varepsilon r}$ will be of substantial importance both in Lemma 3.6 and in Lemma 3.8.

Lemma 3.5 Let $\kappa \in \mathbb{R}$ and $0<\chi<\mu$, and assume that (1.4), (1.5) and (1.6)-(1.8) hold with some $K>0, K_{1}>0$ and $K_{2}>0$. Then there exist $t_{0} \in(0,1)$ and $C>0$ such that for any choice of $\varepsilon \in(0,1)$,

$$
\left|v_{\varepsilon r}(r, t)\right| \leq C r^{1-n} \cdot \frac{\left[\phi^{-1}\left(\frac{1}{t}\right)\right]^{n}}{t} \quad \text { for all } r \in(0, R) \text { and each } t \in\left(0, t_{0}\right) .
$$

Proof As on the right-hand side of (2.13), we can estimate

$$
\begin{aligned}
\frac{1}{n} m_{\varepsilon}(t) r^{n}-\int_{0}^{r} \rho^{n-1} u_{\varepsilon}(\rho, t) \mathrm{d} \rho \geq & -\int_{0}^{R} \rho^{n-1} u_{\varepsilon}(\rho, t) \mathrm{d} \rho \\
= & -\frac{1}{n\left|B_{1}(0)\right|} \cdot \int_{\Omega} u_{\varepsilon}(\cdot, t) \\
& \quad \text { for all } r \in(0, R), t>0 \text { and } \varepsilon \in(0,1),
\end{aligned}
$$

thanks to Lemma 2.5 this directly results from Lemma 3.3.

\subsection{Approaching initial traces in $\left(W_{0}^{2,1}\left(\backslash \bar{B}_{\delta}(0)\right)\right)^{\star}$}

Through (2.13), the information gained in Lemmas 3.3 and 3.4 now provides regularity properties of the gradient $\nabla v_{\varepsilon}$ that are sufficient for the derivation of the following statement on compactness in appropriately large dual spaces.

Lemma 3.6 Let $\kappa \in \mathbb{R}$ and $0<\chi<\mu$, and suppose that (1.4) and (1.5) hold as well as (1.6)-(1.9) with some positive constants $K, K_{1}$ and $K_{2}$. Then for each $\delta \in(0, R)$ and any $T>1$,

$$
\left(u_{\varepsilon}\right)_{\varepsilon \in(0,1)} \text { is relatively compact in } C^{0}\left([0, T] ;\left(W_{0}^{2,1}\left(\Omega \backslash \bar{B}_{\delta}(0)\right)\right)^{\star}\right) .
$$

Proof For fixed $\delta \in(0, R)$, Lemma 3.2 together with (1.6) allows us to fix $c_{1}=c_{1}(\delta)>0$ such that

$$
u_{\varepsilon} \leq c_{1} \quad \text { in }\left(\Omega \backslash \bar{B}_{\delta}(0)\right) \times(0, \infty) \quad \text { for all } \varepsilon \in(0,1),
$$

and Lemma 3.5 in conjunction with Lemma 2.6 states that if we let $t_{0} \in(0,1)$ be as given by Lemma 3.5, and define

$$
f(t):= \begin{cases}\frac{\left[\phi^{-1}\left(\frac{1}{t}\right)\right]^{n}}{t}, & t \in\left(0, t_{0}\right), \\ 1, & t \geq t_{0},\end{cases}
$$

then for all $T>1$ we can find $c_{2}=c_{2}(\delta, T)>0$ fulfilling

$$
\left|\nabla v_{\varepsilon}\right| \leq c_{2} f(t) \quad \text { in }\left(\Omega \backslash \bar{B}_{\delta}(0)\right) \times(0, T) \quad \text { for all } \varepsilon \in(0,1) .
$$


For arbitrary $t \in(0, T)$ and $\psi \in C_{0}^{\infty}\left(\Omega \backslash \bar{B}_{\delta}(0)\right)$, by going back to (2.6) we can thus use (3.31) and (3.33) to estimate

$$
\begin{aligned}
\left|\int_{\Omega} u_{\varepsilon t}(x, t) \psi(x) \mathrm{d} x\right|= & \left|\int_{\Omega} u_{\varepsilon} \Delta \psi+\chi \int_{\Omega} u_{\varepsilon} \nabla v_{\varepsilon} \cdot \nabla \psi+\kappa \int_{\Omega} u_{\varepsilon} \psi-\mu \int_{\Omega} u_{\varepsilon}^{2} \psi\right| \\
\leq & c_{1}\|\Delta \psi\|_{L^{1}(\Omega)}+c_{1} c_{2} \chi f(t)\|\nabla \psi\|_{L^{1}(\Omega)}+c_{1}|\kappa| \cdot\|\psi\|_{L^{1}(\Omega)} \\
& +c_{1}^{2} \mu\|\psi\|_{L^{1}(\Omega)}
\end{aligned}
$$

for all $\varepsilon \in(0,1)$, whence by completion we obtain that with some $c_{3}=c_{3}(\delta, T)>0$,

$$
\left\|u_{\varepsilon t}(\cdot, t)\right\|_{\left(W_{0}^{2,1}\left(\Omega \backslash \bar{B}_{\delta}(0)\right)\right)^{\star}} \leq c_{3} \cdot(f(t)+1) \quad \text { for all } t \in(0, T) \text { and any } \varepsilon \in(0,1) .
$$

In particular, this entails that whenever $t \in[0, T]$ and $s \in[0, t]$,

$$
\begin{aligned}
\left\|u_{\varepsilon}(\cdot, t)-u_{\varepsilon}(\cdot, s)\right\|_{\left(W_{0}^{2,1}\left(\Omega \backslash \bar{B}_{\delta}(0)\right)\right)^{\star}} & =\left\|\int_{s}^{t} u_{\varepsilon t}(\cdot, \sigma) \mathrm{d} \sigma\right\|_{\left(W_{0}^{2,1}\left(\Omega \backslash \bar{B}_{\delta}(0)\right)\right)^{\star}} \\
& \leq c_{3} \int_{s}^{t}(f(\sigma)+1) \mathrm{d} \sigma \quad \text { for all } \varepsilon \in(0,1),(3.34)
\end{aligned}
$$

where we note that since $f+1$ belongs to $L^{1}((0, T))$ due to (3.32) and Lemma 3.4, the uniform continuity of $[0, T] \ni \widehat{t} \mapsto \int_{0}^{\widehat{t}}(f(\sigma)+1) \mathrm{d} \sigma$ thereby implied ensures that

$$
\sup _{\substack{0 \leq s \leq t \leq T \\|s-t|<\eta}} \int_{s}^{t}(f(\sigma)+1) \mathrm{d} \sigma \rightarrow 0 \quad \text { as } \eta \searrow 0 .
$$

Accordingly, (3.34) entails that $\left(u_{\varepsilon}\right)_{\varepsilon \in(0,1)}$ is equi-continuous on $[0, T]$ as a family of $\left(W_{0}^{2,1}\left(\Omega \backslash \bar{B}_{\delta}(0)\right)\right)^{\star}$-valued functions, so that since furthermore, for each fixed $t \in[0, T]$, $\left(u_{\varepsilon}(\cdot, t)\right)_{\varepsilon \in(0,1)}$ is bounded in $L^{\infty}\left(\Omega \backslash \bar{B}_{\delta}(0)\right) \hookrightarrow \hookrightarrow\left(W_{0}^{2,1}\left(\Omega \backslash \bar{B}_{\delta}(0)\right)\right)^{\star}$ by (3.31), the claimed compactness property (3.30) becomes a consequence of the Arzelà-Ascoli theorem.

A natural consequence of the latter completes the following key step in our reasoning.

Lemma 3.7 Assume that $\kappa \in \mathbb{R}$ and $0<\chi<\mu$, and suppose that (1.4) and (1.5) hold as well as (1.6)-(1.9) with some positive constants $K, K_{1}$ and $K_{2}$. Then the limit function $u$ obtained in Lemma 2.7 has the property that for all $\delta \in(0, R)$,

$$
u(\cdot, t) \rightarrow u_{0} \quad \text { in }\left(W_{0}^{2,1}\left(\Omega \backslash \bar{B}_{\delta}(0)\right)\right)^{\star} \quad \text { as } t \searrow 0 .
$$

Proof This is immediately implied by Lemma 3.6 when combined with Lemma 2.6 and the fact that $u_{\varepsilon}(\cdot, 0) \rightarrow u_{0}$ in $\left.L^{\infty}\left(\Omega \backslash \bar{B}_{\delta}(0)\right)\right)$ as $\varepsilon \searrow 0$ according to (2.5).

\subsection{Additional consequences of uniform mass integrability on regularity near $t=0$}

Let us now make sure that outside the spatial origin the upper bound for $u_{\varepsilon}$ from Lemma 3.2, together with the mass control implied by Lemmas 3.3 and 3.4, actually implies further compactness properties of $\left(u_{\varepsilon}(\cdot, t)\right)_{t \in(0,1)}$. By means of a standard testing procedure involving appropriate localization, Lemma 3.5 together with Lemma 3.2 indeed entails the following. 
Lemma 3.8 Assume that $\kappa \in \mathbb{R}$ and $0<\chi<\mu$, and suppose that (1.4) and (1.5) are valid with $K>0$ and $\phi$ fulfilling (1.6) and (1.7) with some $K_{1}>0$. Then for each $\delta \in(0, R)$ and any $T>0$ one can find $C(\delta, T)>0$ such that

$$
\int_{0}^{\mathrm{T}} \int_{\Omega \backslash B_{\delta}(0)}\left|\nabla u_{\varepsilon}(x, t)\right|^{2} \mathrm{~d} x \leq C(\delta, T) \quad \text { for all } \varepsilon \in(0,1) .
$$

Proof Given $\delta \in(0, R)$ and $T>0$, we once more invoke Lemma 3.2, (1.6), Lemmas 2.6, 3.4 and 3.5 to find $c_{1}=c_{1}(\delta)>0, c_{2}=c_{2}(\delta, T)>0$ and $f \in L^{1}((0, T))$ such that

$$
u_{\varepsilon} \leq c_{1} \quad \text { in }\left(\Omega \backslash B_{\frac{\delta}{2}}(0)\right) \times(0, \infty) \quad \text { for all } \varepsilon \in(0,1)
$$

and

$$
\left|\nabla v_{\varepsilon}\right| \leq c_{2} f(t) \quad \text { in }\left(\Omega \backslash B_{\frac{\delta}{2}}(0)\right) \times(0, T) \quad \text { for all } \varepsilon \in(0,1) .
$$

We then pick a cutoff function $\zeta \in C^{\infty}(\bar{\Omega})$ such that $0 \leq \zeta \leq 1$ in $\Omega, \zeta \equiv 1$ in $\Omega \backslash B_{\delta}(0)$ and $\zeta \equiv 0$ in $B_{\frac{\delta}{2}}(0)$, and test the first equation in (2.6) against $\zeta u_{\varepsilon}$ in a standard manner to see that for each $\varepsilon \in(0,1)$,

$$
\begin{aligned}
\frac{1}{2} \frac{d}{\mathrm{~d} t} \int_{\Omega} \zeta(x) u_{\varepsilon}^{2}(x, t) \mathrm{d} x= & \int_{\Omega} \zeta u_{\varepsilon} \cdot\left\{\Delta u_{\varepsilon}-\chi \nabla \cdot\left(u_{\varepsilon} \nabla v_{\varepsilon}\right)+\kappa u_{\varepsilon}-\mu u_{\varepsilon}^{2}\right\} \\
= & -\int_{\Omega} \zeta\left|\nabla u_{\varepsilon}\right|^{2}-\int_{\Omega} u_{\varepsilon} \nabla u_{\varepsilon} \cdot \nabla \zeta \\
& +\chi \int_{\Omega} \zeta u_{\varepsilon} \nabla u_{\varepsilon} \cdot \nabla v_{\varepsilon}+\chi \int_{\Omega} u_{\varepsilon}^{2} \nabla v_{\varepsilon} \cdot \nabla \zeta \\
& +\kappa \int_{\Omega} \zeta u_{\varepsilon}^{2}-\mu \int_{\Omega} \zeta u_{\varepsilon}^{3} \quad \text { for all } t>0
\end{aligned}
$$

Here upon another integration by parts, we can use (3.37) to estimate

$$
-\int_{\Omega} u_{\varepsilon} \nabla u_{\varepsilon} \cdot \nabla \zeta=\frac{1}{2} \int_{\Omega} u_{\varepsilon}^{2} \Delta \zeta \leq \frac{c_{1}^{2}}{2}\|\Delta \zeta\|_{L^{1}(\Omega)} \quad \text { for all } t>0,
$$

and similarly we may treat the third summand on the right of (3.39) to find that due to the second equation from (2.6) and our assumption that $\mu>\chi>\frac{\chi}{2}$,

$$
\begin{aligned}
& \chi \int_{\Omega} \zeta u_{\varepsilon} \nabla u_{\varepsilon} \cdot \nabla v_{\varepsilon}+\chi u_{\varepsilon}^{2} \nabla v_{\varepsilon} \cdot \nabla \zeta-\mu \int_{\Omega} \zeta u_{\varepsilon}^{3} \\
& =-\frac{\chi}{2} \int_{\Omega} \zeta u_{\varepsilon}^{2} \Delta v_{\varepsilon}+\frac{\chi}{2} \int_{\Omega} u_{\varepsilon}^{2} \nabla v_{\varepsilon} \cdot \nabla \zeta-\mu \int_{\Omega} \zeta u_{\varepsilon}^{3} \\
& \quad=-\frac{\chi}{2} m_{\varepsilon}(t) \cdot \int_{\Omega} \zeta u_{\varepsilon}^{2}-\left(\mu-\frac{\chi}{2}\right) \int_{\Omega} \zeta u_{\varepsilon}^{3}+\frac{\chi}{2} \int_{\Omega} u_{\varepsilon}^{2} \nabla v_{\varepsilon} \cdot \nabla \zeta \\
& \quad \leq \frac{\chi}{2} \int_{\Omega} u_{\varepsilon}^{2} \nabla v_{\varepsilon} \cdot \nabla \zeta \\
& \quad \leq \frac{\chi}{2} c_{1}^{2} c_{2}\|\nabla \zeta\|_{L^{1}(\Omega)} \cdot f(t) \quad \text { for all } t \in(0, T) .
\end{aligned}
$$

As clearly, also by (3.37),

$$
\int_{\Omega} \zeta u_{\varepsilon}^{2} \leq c_{1}^{2}|\Omega| \quad \text { for all } t>0
$$


from (3.39)-(3.41) we infer the existence of $c_{3}=c_{3}(\delta, T)>0$ such that for arbitrary $\varepsilon \in(0,1)$,

$$
\frac{d}{\mathrm{~d} t} \int_{\Omega} \zeta u_{\varepsilon}^{2}+2 \int_{\Omega} \zeta\left|\nabla u_{\varepsilon}\right|^{2} \leq c_{3} \cdot(f(t)+1) \quad \text { for all } t \in(0, T),
$$

which on integration yields

$$
\int_{\Omega} \zeta u_{\varepsilon}^{2}(\cdot, T)+2 \int_{0}^{\mathrm{T}} \int_{\Omega} \zeta\left|\nabla u_{\varepsilon}\right|^{2} \leq \int_{\Omega} \zeta u_{0 \varepsilon}^{2}+c_{3} \int_{0}^{\mathrm{T}}(f(t)+1) \mathrm{d} t \quad \text { for all } \varepsilon \in(0,1) \text {. }
$$

Since (2.4) and (1.5) along with the fact that $\operatorname{supp} \zeta \subset \bar{\Omega} \backslash B_{\frac{\delta}{2}}(0)$ enforce that $\sup _{\varepsilon \in(0,1)} \int_{\Omega} \zeta u_{0 \varepsilon}^{2}$ is finite, and since $f+1$ belongs to $L^{1}((0, T))$, recalling that $\zeta$ is nonnegative and satisfies $\zeta \equiv 1$ in $\Omega \backslash B_{\delta}(0)$ we obtain (3.36) as a consequence of (3.43).

One final regularity argument, now using a localization slightly more subtle and thereby favorably cooperating with our knowledge on radial symmetry and monotonicity, yields even certain temporally uniform bounds for $\nabla u_{\varepsilon}$ in annular regions.

Lemma 3.9 Let $\kappa \in \mathbb{R}$ and $0<\chi<\mu$, and let (1.4)-(1.7) be satisfied with some $K>0$ and $K_{1}>0$. Then for any $\delta \in(0, R)$ and $T>0$ there exists $C(\delta, T)>0$ such that whenever $\varepsilon \in(0,1)$,

$$
\int_{\Omega \backslash B_{\delta}(0)}\left|\nabla u_{\varepsilon}(x, t)\right|^{2} \mathrm{~d} x \leq C(\delta, T) \quad \text { for all } t \in(0, T) .
$$

Proof For fixed $\delta \in(0, R)$, we choose a cutoff function $\zeta \in C^{\infty}(\bar{\Omega})$ such that again $\zeta \equiv 0$ in $B_{\frac{\delta}{2}}(0)$ and $\zeta \equiv 1$ in $\Omega \backslash B_{\delta}(0)$, but such that now, in contrast to the requirements from the proof of Lemma 3.8, $\zeta$ additionally is radially symmetric and nondecreasing with respect to $r=|x| \in[0, R]$. Then upon several integrations by parts, we obtain from (2.8) that for all $\varepsilon \in(0,1)$,

$$
\begin{aligned}
\frac{1}{2} \frac{d}{\mathrm{~d} t} \int_{\Omega} \zeta^{2}(x)\left|\nabla u_{\varepsilon}(x, t)\right|^{2} \mathrm{~d} x= & \int_{\Omega} \zeta^{2} \nabla u_{\varepsilon} \cdot \nabla\left\{\Delta u_{\varepsilon}-\chi \nabla u_{\varepsilon} \cdot \nabla v_{\varepsilon}-\chi m_{\varepsilon}(t) u_{\varepsilon}\right. \\
+ & \left.\kappa u_{\varepsilon}-(\mu-\chi) u_{\varepsilon}^{2}\right\} \\
= & -\int_{\Omega} \zeta^{2}\left|\Delta u_{\varepsilon}\right|^{2}-2 \int_{\Omega} \zeta\left(\nabla u_{\varepsilon} \cdot \nabla \zeta\right) \Delta u_{\varepsilon} \\
& -\chi \int_{\Omega} \zeta^{2} \nabla u_{\varepsilon} \cdot \nabla\left(\nabla u_{\varepsilon} \cdot \nabla v_{\varepsilon}\right)-\chi m_{\varepsilon}(t) \int_{\Omega} \zeta^{2}\left|\nabla u_{\varepsilon}\right|^{2} \\
& +\kappa \int_{\Omega} \zeta^{2}\left|\nabla u_{\varepsilon}\right|^{2}-2(\mu-\chi) \int_{\Omega} \zeta^{2} u_{\varepsilon}\left|\nabla u_{\varepsilon}\right|^{2} \quad \text { for all } t>0,
\end{aligned}
$$

where by Young's inequality,

$$
-2 \int_{\Omega} \zeta\left(\nabla u_{\varepsilon} \cdot \nabla \zeta\right) \Delta u_{\varepsilon} \leq \int_{\Omega} \zeta^{2}\left|\Delta u_{\varepsilon}\right|^{2}+\int_{\Omega}|\nabla \zeta|^{2}\left|\nabla u_{\varepsilon}\right|^{2} \quad \text { for all } t>0 .
$$

In the crucial third summand on the right of (3.45), we resort to the radial notation again to see upon further integration by parts that thanks to the boundary condition $u_{\varepsilon r}(R, \cdot) \equiv 0$ and the second equation in (2.6),

$$
-\int_{0}^{R} r^{n-1} \zeta^{2}(r) u_{\varepsilon r} \cdot\left(u_{\varepsilon r} v_{\varepsilon r}\right)_{r} \mathrm{~d} r
$$




$$
\begin{aligned}
= & -\int_{0}^{R} r^{n-1} \zeta^{2}(r) u_{\varepsilon r} u_{\varepsilon r r} v_{\varepsilon r} \mathrm{~d} r-\int_{0}^{R} r^{n-1} \zeta^{2}(r) u_{\varepsilon r}^{2} v_{\varepsilon r r} \mathrm{~d} r \\
= & \frac{1}{2} \int_{0}^{R} r^{n-1} \zeta^{2}(r) u_{\varepsilon r}^{2}\left(v_{\varepsilon r r}+\frac{n-1}{r} v_{\varepsilon r}\right) \mathrm{d} r+\int_{0}^{R} r^{n-1} \zeta(r) \zeta_{r}(r) u_{\varepsilon r}^{2} v_{\varepsilon r} \mathrm{~d} r \\
& -\int_{0}^{R} r^{n-1} \zeta^{2}(r) u_{\varepsilon r}^{2} v_{\varepsilon r r} \mathrm{~d} r \\
= & -\frac{1}{2} \int_{0}^{R} r^{n-1} \zeta^{2}(r) u_{\varepsilon r}^{2}\left(v_{\varepsilon r r}+\frac{n-1}{r} v_{\varepsilon r}\right) \mathrm{d} r+\int_{0}^{R} r^{n-1} \zeta(r) \zeta_{r}(r) u_{\varepsilon r}^{2} v_{\varepsilon r} \mathrm{~d} r \\
& +(n-1) \int_{0}^{R} r^{n-2} \zeta^{2}(r) u_{\varepsilon r}^{2} v_{\varepsilon r} \mathrm{~d} r \\
= & -\frac{1}{2} m_{\varepsilon}(t) \int_{0}^{R} r^{n-1} \zeta^{2}(r) u_{\varepsilon r}^{2} \mathrm{~d} r+\frac{1}{2} \int_{0}^{R} r^{n-1} \zeta^{2}(r) u_{\varepsilon} u_{\varepsilon r}^{2} \mathrm{~d} r \\
& +\int_{0}^{R} r^{n-1} \zeta(r) \zeta_{r}(r) u_{\varepsilon r}^{2} v_{\varepsilon r} \mathrm{~d} r+(n-1) \int_{0}^{R} r^{n-2} \zeta^{2}(r) u_{\varepsilon r}^{2} v_{\varepsilon r} \mathrm{~d} r \quad \text { for all } t>0 .
\end{aligned}
$$

Since $u_{\varepsilon r} \leq 0$ and $v_{\varepsilon r} \leq 0$ by Lemma 2.4 and Lemma 2.5, our requirement on upward radial monotonicity of $\zeta$ thus ensures that, besides the fourth last and the last, also the second last summand herein is nonpositive for all $\varepsilon \in(0,1)$ and $t>0$. Therefore,

$$
-\chi \int_{\Omega} \zeta^{2} \nabla u_{\varepsilon} \cdot \nabla\left(\nabla u_{\varepsilon} \cdot \nabla v_{\varepsilon}\right) \leq \frac{\chi}{2} \int_{\Omega} \zeta^{2} u_{\varepsilon}\left|\nabla u_{\varepsilon}\right|^{2} \quad \text { for all } t>0
$$

whence (3.45) and (3.46) along with our assumption that $\mu>\chi$ imply that

$$
\begin{aligned}
& \frac{1}{2} \frac{d}{\mathrm{~d} t} \int_{\Omega} \zeta^{2}\left|\nabla u_{\varepsilon}\right|^{2} \leq \int_{\Omega}|\nabla \zeta|^{2}\left|\nabla u_{\varepsilon}\right|^{2}+\kappa_{+} \int_{\Omega} \zeta^{2}\left|\nabla u_{\varepsilon}\right|^{2}+\frac{\chi}{2} \int_{\Omega} \zeta^{2} u_{\varepsilon}\left|\nabla u_{\varepsilon}\right|^{2} \\
& \text { for all } t>0 .
\end{aligned}
$$

Again relying on Lemma 3.2 and (1.6), we thus conclude that for any such $\delta$ and each $T>0$ we can find $c_{1}=c_{1}(\delta, T)>0$ fulfilling

$$
\frac{d}{\mathrm{~d} t} \int_{\Omega} \zeta^{2}\left|\nabla u_{\varepsilon}\right|^{2} \leq c_{1} \int_{\Omega \backslash B_{\frac{\delta}{2}}(0)}\left|\nabla u_{\varepsilon}\right|^{2} \quad \text { for all } t \in(0, T) \text { and any } \varepsilon \in(0,1)
$$

and that accordingly, for each $\varepsilon \in(0,1)$,

$$
\int_{\Omega} \zeta^{2}(x)\left|\nabla u_{\varepsilon}(x, t)\right|^{2} \mathrm{~d} x \leq \int_{\Omega} \zeta^{2}(x)\left|\nabla u_{0 \varepsilon}(x)\right|^{2} \mathrm{~d} x+c_{1} \int_{0}^{\mathrm{T}} \int_{\Omega \backslash B_{\frac{\delta}{2}}(0)}\left|\nabla u_{\varepsilon}\right|^{2}
$$

for all $t \in(0, T)$.

In view of (2.5), the outcome of Lemma 3.8 therefore warrants validity of (3.44) with some suitably large $C(\delta, T)>0$.

\subsection{Locally uniform initial trace attainment: proof of the main results}

One last time making use of radial symmetry, from Lemma 3.9 and the compactness of the embedding $W^{1,2}((\delta, R)) \hookrightarrow C^{0}([\delta, R])$ for $\delta \in(0, R)$, we can finally infer the following. 
Lemma 3.10 Assume that $\kappa \in \mathbb{R}$ and $0<\chi<\mu$, and suppose that (1.4) and (1.5) as well as (1.6)-(1.9) hold with some positive constants $K, K_{1}$ and $K_{2}$. Then the function $u$ given by Lemma 2.7 belongs to $\left.C^{0}(\bar{\Omega} \backslash\{0\}) \times[0, \infty)\right)$ and satisfies

$$
u(\cdot, t) \rightarrow u_{0} \quad \text { in } C_{\mathrm{loc}}^{0}(\bar{\Omega} \backslash\{0\}) \quad \text { as } t \searrow 0 .
$$

Proof As $u_{0}$ is continuous in $\bar{\Omega} \backslash\{0\}$, in view of the regularity properties asserted by Lemma 2.7, it is sufficient to verify (3.47). In fact, if this was false then there would exist $\delta \in(0, R)$ and $\left(t_{k}\right)_{k \in \mathbb{N}} \subset(0,1)$ such that $t_{k} \searrow 0$ as $k \rightarrow \infty$ and

$$
\inf _{k \in \mathbb{N}}\left\|u\left(\cdot, t_{k}\right)-u_{0}\right\|_{C^{0}\left(\bar{\Omega} \backslash B_{\delta}(0)\right)}>0 .
$$

On the other hand, combining Lemma 3.9 with the convergence statement from Lemma 2.7 provides $c_{1}>0$ such that, again in radial coordinates, we have

$$
\int_{\delta}^{R} r^{n-1} u_{r}^{2}(r, t) \mathrm{d} r \leq c_{1} \quad \text { for all } t \in(0,1)
$$

and hence, by the Cauchy-Schwarz inequality,

$$
\begin{aligned}
\left|u\left(r_{2}, t\right)-u\left(r_{1}, t\right)\right| & =\left|\int_{r_{1}}^{r_{2}} u_{r}(r, t) \mathrm{d} r\right| \\
& \leq\left\{\int_{r_{1}}^{r_{2}} r^{n-1} u_{r}^{2}(r, t) \mathrm{d} r\right\}^{\frac{1}{2}} \cdot\left\{\int_{r_{1}}^{r_{2}} r^{1-n} \mathrm{~d} r\right\}^{\frac{1}{2}} \\
& \leq c_{1}^{\frac{1}{2}} \delta^{\frac{1-n}{2}}\left(r_{2}-r_{1}\right)^{\frac{1}{2}} \quad \text { for all } r_{1} \in[\delta, R], r_{2} \in\left[r_{1}, R\right] \text { and } t \in(0,1) .
\end{aligned}
$$

Together with Lemma 3.2 and (1.6), by the Arzelà-Ascoli theorem this equi-continuity property warrants that $\left(u\left(\cdot, t_{k}\right)\right)_{k \in \mathbb{N}}$ is relatively compact in $C^{0}\left(\bar{\Omega} \backslash B_{\delta}(0)\right)$, so that for some subsequence $\left(t_{k_{j}}\right)_{j \in \mathbb{N}}$ of $\left(t_{k}\right)_{k \in \mathbb{N}}$ we can find $z \in C^{0}\left(\bar{\Omega} \backslash B_{\delta}(0)\right)$ such that $u\left(\cdot, t_{k_{j}}\right) \rightarrow z$ in $C^{0}\left(\bar{\Omega} \backslash B_{\delta}(0)\right)$ as $j \rightarrow \infty$. But since from Lemma 3.7 we already know that $u(\cdot, t) \rightarrow u_{0}$ in $\left(W_{0}^{2,1}\left(\Omega \backslash \bar{B}_{\delta}(0)\right)\right)^{\star}$ as $t \searrow 0$, this necessarily implies that $z=u_{0}$ and thereby contradicts (3.48).

Proving our main results thereby essentially reduces to collecting tesserae:

Proof of Theorem 1.1 We only need to combine the results from Lemma 2.7, 3.3 and 3.4 with the convergence statement asserted by Lemma 3.10.

Proof of Corollary 1.2 Setting $\phi(r):=e^{\lambda r^{-\alpha}}, r>0$, we immediately see that (1.5) and (1.6) hold, and computing

$$
\begin{aligned}
\phi^{\prime}(r) & =-\alpha \lambda r^{-\alpha-1} e^{\lambda r^{-\alpha}} \text { and } \phi^{\prime \prime}(r)=\alpha^{2} \lambda^{2} r^{-2 \alpha-2} e^{\lambda r^{-\alpha}}+\alpha(\alpha+1) \lambda r^{-\alpha-2} e^{\lambda r^{-\alpha}}, \\
r & >0,
\end{aligned}
$$

we see that

$$
\frac{\phi^{\prime \prime}(r)}{\phi^{2}(r)}=\alpha^{2} \lambda^{2} r^{-2 \alpha-2} e^{-\lambda r^{-\alpha}}+\alpha(\alpha+1) \lambda r^{-\alpha-2} e^{-\lambda r^{-\alpha}} \rightarrow 0 \quad \text { as } r \searrow 0,
$$

which implies (1.7) for some suitably large $K_{1}>0$. Likewise, (1.8) can be achieved upon observing that

$$
\frac{\phi(2 r)}{r^{n} \phi(r)}=r^{-n} e^{-\left(1-2^{-\alpha}\right) \lambda r^{-\alpha}} \rightarrow 0 \quad \text { as } r \searrow 0,
$$

whereas (1.9) is a direct consequence of the hypothesis (1.15). 
Acknowledgements The author acknowledges support of the Deutsche Forschungsgemeinschaft in the context of the project Analysis of chemotactic cross-diffusion in complex frameworks.

\section{References}

1. Aida, M., Tsujikawa, T., Efendiev, M., Yagi, A., Mimura, M.: Lower estimate of the attractor dimension for a chemotaxis growth system. J. Lond. Math. Soc. 74, 453-474 (2006)

2. Bedrossian, J., Masmoudi, N.: Existence, uniqueness and Lipschitz dependence for Patlak-Keller-Segel and Navier-Stokes in $\mathbb{R}^{2}$ with measure-valued initial data. Arch. Ration. Mech. Anal. 214, 717-801 (2014)

3. Bellomo, N., Winkler, M.: Finite-time blow-up in a degenerate chemotaxis system with flux limitation. Trans. Am. Math. Soc. Ser. B 4, 31-67 (2017)

4. Biler, P.: The Cauchy problem and self-similar solutions for a nonlinear parabolic equation. Stud. Math. 114, 181-205 (1995)

5. Biler, P.: Local and global solvability of some parabolic systems modelling chemotaxis. Adv. Math. Sci. Appl. 8, 715-743 (1998)

6. Blanchet, A., Carrillo, J.A., Kinderlehrer, D., Kowalczyk, M., Laurençot, Ph, Lisini, S.: A hybrid variational principle for the Keller-Segel system in $\mathbb{R}^{2}$. ESAIM Math. Model. Numer. Anal. 49, 1553-1576 (2015)

7. Blanchet, A., Dolbeault, J., Perthame, B.: Two-dimensional Keller-Segel model: optimal critical mass and qualitative properties of the solutions. Electron. J. Differ. Eq. 2006(44), 32 (2006)

8. Calvez, V., Carrillo, J.A.: Volume effects in the Keller-Segel model: energy estimates preventing blow-up. J. Math. Pures Appl. 86, 155-175 (2006)

9. Cao, X.: Large time behavior in the logistic Keller-Segel model via maximal Sobolev regularity. Discrete Contin. Dyn. Syst. Ser. B 22, 3369-3378 (2017)

10. Cao, X., Lankeit, J.: Global classical small-data solutions for a three-dimensional chemotaxis NavierStokes system involving matrix-valued sensitivities. Calc. Var. Part. Differ. Eq. 55, 39 (2016). (Paper No. 107)

11. Chaplain, M.A.J., Lolas, G.: Mathematical modelling of cancer invasion of tissue: the role of the urokinase plasminogen activation system. Math. Mod. Methods Appl. Sci. 15, 1685-1734 (2005)

12. Cieślak, T., Stinner, C.: New critical exponents in a fully parabolic quasilinear Keller-Segel and applications to volume filling models. J. Differ. Eq. 258(6), 2080-2113 (2015)

13. Dolbeault, J., Schmeiser, C.: The two-dimensional Keller-Segel model after blow-up. Discrete Contin. Dyn. Syst. 25, 109-121 (2009)

14. Herrero, M.A., Velázquez, J.J.L.: A blow-up mechanism for a chemotaxis model. Ann. Scu. Norm. Sup. Pisa Cl. Sci. 24, 633-683 (1997)

15. Hillen, T., Painter, K.: A user's guide to PDE models for chemotaxis. J. Math. Biol. 58, 183-217 (2009)

16. Jäger, W., Luckhaus, S.: On explosions of solutions to a system of partial differential equations modelling chemotaxis. Trans. Am. Math. Soc. 329, 819-824 (1992)

17. Kang, K., Stevens, A.: Blowup and global solutions in a chemotaxis-growth system. Nonlinear Anal. 135, 57-72 (2016)

18. Keller, E.F., Segel, L.A.: Initiation of slime mold aggregation viewed as an instability. J. Theor. Biol. 26, 399-415 (1970)

19. Ladyzenskaja, O.A., Solonnikov, V.A., Ural'ceva, N.N.: Linear and Quasi-Linear Equations of Parabolic Type, vol. 23. Amer. Math. Soc. Transl., Providence (1968)

20. Lankeit, J.: Chemotaxis can prevent thresholds on population density. Discrete Contin. Dyn. Syst. Ser. B 20, 1499-1527 (2015)

21. Lankeit, J.: Eventual smoothness and asymptotics in a three-dimensional chemotaxis system with logistic source. J. Differ. Eq. 258, 1158-1191 (2015)

22. Lankeit, J.: Infinite time blow-up of many solutions to a general quasilinear parabolic-elliptic Keller-Segel system. Discrete Contin. Dyn. Syst. Ser. (to appear)

23. Liu, D., Tao, Y.: Boundedness in a chemotaxis system with nonlinear signal production. Appl. Math. J. Chin. Univ. Ser. B 31, 379-388 (2016)

24. Luckhaus, S., Sugiyama, Y., Velázquez, J.J.L.: Measure valued solutions of the 2D Keller-Segel system. Arch. Ration. Mech. Anal. 206, 31-80 (2012)

25. Nagai, T.: Blow-up of radially symmetric solutions to a chemotaxis system. Adv. Math. Sci. Appl. 5, 581-601 (1995) 
26. Nagai, T.: Blowup of nonradial solutions to parabolic-elliptic systems modeling chemotaxis in twodimensional Domains. J. Inequal. Appl. 6, 37-55 (2001)

27. Nagai, T., Senba, T., Suzuki, T.: Chemotactic collapse in a parabolic system of mathematical biology. Hiroshima Math. J. 30, 463-497 (2000)

28. Osaki, K., Tsujikawa, T., Yagi, A., Mimura, M.: Exponential attractor for a chemotaxis-growth system of equations. Nonlinear Anal. 51, 119-144 (2002)

29. Osaki, K., Yagi, A.: Finite dimensional attractor for one-dimensional Keller-Segel equations. Funkc. Ekvacioj 44, 441-469 (2001)

30. Painter, K.J.: Mathematical models for chemotaxis and their applications in self-organisation phenomena. J. Theor. Biol. (to appear)

31. Painter, K.J., Hillen, T.: Spatio-temporal chaos in a chemotaxis model. Physica D 240, 363-375 (2011)

32. Painter, K.J., Maini, P.K., Othmer, H.G.: Complex spatial patterns in a hybrid chemotaxis reactiondiffusion model. J. Math. Biol. 41(4), 285-314 (2000)

33. Raczynski, A.: Stability property of the two-dimensional Keller-Segel model. Asymptot. Anal. 61, 35-59 (2009)

34. Senba, T., Suzuki, T.: Weak solutions to a parabolic-elliptic system of chemotaxis. J. Funct. Anal. 191, 17-51 (2002)

35. Szymańska, Z., Morales Rodrigo, C., Lachowicz, M., Chaplain, M.A.: Mathematical modelling of cancer invasion of tissue: the role and effect of nonlocal interactions. Math. Mod. Methods Appl. Sci. 19, 257-281 (2009)

36. Tao, Y., Winkler, M.: Boundedness in a quasilinear parabolic-parabolic Keller-Segel system with subcritical sensitivity. J. Differ. Eq. 252(1), 692-715 (2012)

37. Tao, Y., Winkler, M.: Critical mass for infinite-time aggregation in a chemotaxis model with indirect signal production. J. Eur. Math. Soc. 19, 3641-3678 (2017)

38. Tello, J.I., Winkler, M.: A chemotaxis system with logistic source. Commun. Part. Differ. Eq. 32(6), 849-877 (2007)

39. Waldeland, J.O., Evje, S.: A multiphase model for exploring tumor cell migration driven by autologous chemotaxis. Chem. Eng. Sci. 191, 268-287 (2018)

40. Winkler, M.: Does a 'volume-filling effect' always prevent chemotactic collapse? Math. Methods Appl. Sci. 33, 12-24 (2010)

41. Winkler, M.: Boundedness in the higher-dimensional parabolic-parabolic chemotaxis system with logistic source. Commun. Part. Differ. Eq. 35, 1516-1537 (2010)

42. Winkler, M.: Finite-time blow-up in the higher-dimensional parabolic-parabolic Keller-Segel system. J. Math. Pures Appl. 100, 748-767 (2013). arXiv:1112.4156v1

43. Winkler, M.: Global asymptotic stability of constant equilibria in a fully parabolic chemotaxis system with strong logistic dampening. J. Differ. Eq. 257, 1056-1077 (2014)

44. Winkler, M.: How far can chemotactic cross-diffusion enforce exceeding carrying capacities? J. Nonlinear Sci. 24, 809-855 (2014)

45. Winkler, M.: Emergence of large population densities despite logistic growth restrictions in fully parabolic chemotaxis systems. Discrete Contin. Dyn. Syst. Ser. B 22, 2777-2793 (2017)

46. Winkler, M.: A critical blow-up exponent in a chemotaxis system with nonlinear signal production. Nonlinearity 31, 2031-2056 (2018)

47. Winkler, M., Djie, K.: Boundedness and finite-time collapse in a chemotaxis system with volume-filling effect. Nonlinear Anal. Theory Methods Appl. 72(2), 1044-1064 (2010)

48. Xiang, T.: How strong a logistic damping can prevent blow-up for the minimal Keller-Segel chemotaxis system? Nonlinear Anal. Real World Appl. 459, 1172-1200 (2018)

Publisher's Note Springer Nature remains neutral with regard to jurisdictional claims in published maps and institutional affiliations. 